\title{
Variaciones al Test de Hodge modificado para la detección de carbapenemasas en Pseudomonas aeruginosa
}

\author{
Variations in the modified Hodge test to detect \\ carbapenemase production in Pseudomonas aeruginosa \\ Ana M. Ocampo-Ríos MSc', Luz M. Giraldo-Gómez MB², \\ Karen L.F. Melo-Álvarez MB2 , Anyela C. Obando-Agreda $M B^{2}$, \\ J. Natalia Jiménez-Quiceno $P h D^{3}$
}

Introducción: la producción de enzimas con actividad hidrolítica frente a carbapenémicos, denominadas carbapenemasas, es uno de los principales mecanismos de resistencia a carbapenémicos en Pseudomonas aeruginosa. El test de Hodge modificado es la prueba fenotípica más empleada para la detección de carbapenemasas; sin embargo, de acuerdo con el CLSI, este método sólo puede emplearse en Enterobacteriáceas ya que presenta limitaciones en Bacilos Gram negativos no fermentadores como Pseudomonas aeruginosa. Objetivo: evaluar la eficacia de variaciones del test de Hodge modificado para la detección de carbapenemasas en aislados de Pseudomonas aeruginosa. Materiales y métodos: se evaluó el desempeño del test 3D y tres variaciones del test de Hodge modificado, tomando como prueba de referencia la detección de los genes de las carbapenemasas KPC, VIM, IMP, NDM y OXA-48 mediante reacción en cadena de la polimerasa. Las variaciones consistieron en emplear: a) agar MacConkey en lugar de Mueller Hinton, b) Klebsiella pneumoniae ATCC 700603 como cepa indicadora sensible a carbapenémicos en lugar de Escherichia coli ATCC 29212 y c) las dos condiciones anteriores simultáneamente. Resultados: de los ensayos evaluados, la tercera variación, que empleó tanto agar MacConkey como la cepa indicadora de Klebsiella pneumoniae ATCC 700603, mostró los mejores valores de sensibilidad y especificidad para la detección de carbapenemasas en Pseudomonas aeruginosa (90,0\% y 85,7\%, respectivamente). Conclusiones: la implementación de las variaciones del test de Hodge modificado podría ser una alternativa económica y de fácil realización para la detección fenotípica de carbapenemasas en Pseudomonas aeruginosa en los laboratorios de Microbiología Clínica.

\footnotetext{
' Microbióloga y Bioanalista, MSc en Microbiología. Profesora, Grupo de Investigación en Microbiología Básica y aplicada -MICROBA-, Escuela de Microbiología. Universidad de Antioquia. Medellín, Colombia.

${ }^{2}$ Estudiante de Microbiología y Bioanálisis, Escuela de Microbiología, Universidad de Antioquia. Medellín, Colombia.

${ }^{3}$ Bacterióloga, MSc y PhD en Ciencias Básicas Biomédicas. Profesora, Grupo de Investigación en Microbiología Básica y aplicada -MICROBA-, Escuela de Microbiología, Universidad de Antioquia. Medellín, Colombia. Correspondencia: Calle 67 N. ${ }^{\circ}$ 53-108, bloque 5, oficina 135, Universidad de Antioquia. Teléfonos 574 21 95497. Correo electrónico: nataliajiudea@gmail.com

Conflicto de intereses: los autores declaran que no tienen conflicto de intereses Medicina \& Laboratorio 2015; 21 : 551-564

Módulo 19 (Investigación), número 42. Editora Médica Colombiana S.A. $2015^{\odot}$

Recibido el 15 de diciembre de 2015; aceptado el 29 de diciembre de 2015
} 
Palabras claves: carbapenemasas, carbapenémicos, detección de carbapenemasas, Pseudomonas aeruginosa, test de Hodge modificado.

Introduction: One of the most important mechanisms of carbapenem resistance in Pseudomonas aeruginosa is the production of carbapenem-hydrolyzing enzymes known as carbapenemases. The modified Hodge test is the most frequently used phenotypic screening test for carbapenemases. Nevertheless, CLSI recommends using modified Hodge test only in members of Enterobacteriaceae, since the test has demonstrated limitations in other Gram-negative bacilli and non-glucose-fermenters as Pseudomonas aeruginosa. Objective: To evaluate the performance of 3D test and three variations of modified Hodge test to detect carbapenemases in Pseudomonas aeruginosa isolates. Materials and methods: The efficacy of 3D test and three variations in modified Hodge test were evaluated taking polymerase chain reaction for carbapenemases KPC, VIM, IMP, NDM and OXA-48 as the gold standard. Variations consisted in using a) MacConckey agar instead of Mueller Hinton, b) Klebsiella pneumoniae ATCC 700603 as indicator carbapenem-sensitive strain instead of Escherichia coli ATCC 29212 and c) last two conditions simultaneously. Results: Of the variations tested, the third assay using both MacConckey agar and Klebsiella pneumoniae ATCC 700603 showed the best sensitivity and specificity (90.0\% and $85.7 \%$, respectively). Conclusions: The implementation of variations in modified Hodge test could be a cheap and easy to perform alternative for phenotypic carbapenemase detection in Pseudomonas aeruginosa in Clinical Microbiology laboratories.

Keywords: Carbapenemases, carbapenems, carbapenemase detection, Pseudomonas aeruginosa, Modified Hodge Test

\section{Ocampo-Ríos AM, Giraldo-Gómez LM, Melo-Álvarez KLF, Obando-Agreda AC, Jiménez-Quiceno JN.} Variaciones al Test de Hodge modificado para la detección de carbapenemasas en Pseudomonas aeruginosa. Medicina \& Laboratorio 2015; 21: 551 -564.

Peudomonas aeruginosa es un bacilo Gram negativo no fermentador que se destaca por ser
un patógeno oportunista, causante de infecciones asociadas a la atención en salud [ I -4]. Esta bacteria es responsable de diversos cuadros clínicos que pueden ir desde infecciones del tracto urinario, la piel y los tejidos blandos hasta infecciones que comprometen la vida del paciente como bacteriemias y neumonías [2]. Gran parte del éxito de esta bacteria se debe a la variedad de los mecanismos de resistencia a los antibióticos que posee tanto intrínsecos como adquiridos, lo cual limita cada vez más las opciones de tratamiento [1,2,5-7]. Los carbapenémicos son los medicamentos de elección para el tratamiento de las infecciones producidas por Pseudomonas aeruginosa multirresistente debido a su amplio espectro y su estabilidad frente a la acción de las $\beta$-lactamasas como las AmpC [8,9]. Sin embargo, en los últimos años, con la emergencia y la frecuencia cada vez mayor de cepas resistentes a los carbapenémicos, se ha complicado el manejo de las infecciones ocasionadas por esta bacteria $[8,10,1 \mathrm{I}]$.

La resistencia a los carbapenémicos en Pseudomonas aeruginosa está mediada principalmente por la presencia de mecanismos cromosómicos como la sobrexpresión de la $\beta$-lactamasa cromosómica AmpC, sumada a la sobreproducción de bombas de expulsión tales como la MexAB-OprM y la impermeabilidad mediada por la pérdida o alteración estructural de la porina OprD $[10,12,13]$. Otro mecanismo de resistencia de gran importancia es la producción de $\beta$-lactamasas con acción hidrolítica frente a los carbapenémicos, conocidas como carbape- 
nemasas [12-17], las cuales confieren resistencia a todos los $\beta$-lactámicos y generalmente se encuentran codificadas en elementos genéticos móviles como los plásmidos y los transposones, lo que le confiere un alto potencial de diseminación [14, I 8-22]. En Pseudomona aeruginosa se han descrito diferentes tipos de carbapenemasas; no obstante, las más frecuentes son las que pertenecen a la clase B (metalo- $\beta$-lactamasas IMP, VIM, SPM, GIM, AIM y DIM) y clase A (KPC), mientras que las de clase $D(O X A)$ se han reportado en menor frecuencia [|4,|8-2 I].

La detección de cepas productoras de carbapenemasas en el laboratorio clínico es de gran importancia para la determinación de los regímenes terapéuticos apropiados y la aplicación de medidas de control de infecciones en miras de contener su diseminación [19,23-26]. El test de Hodge modificado es una prueba fenotípica ampliamente utilizada por los laboratorios de Microbiología Clínica debido a su simplicidad y alta sensibilidad para la detección de carbapenemasas, principalmente de clase A como la KPC $[27,28]$. Sin embargo, hasta el momento el Instituto de Estándares Clínicos y de Laboratorio (CLSI; del inglés, Clinical and Laboratory Standards Institute) sólo recomienda el empleo de esta prueba en bacterias miembros de la familia Enterobacteriaceae [29], ya que ha mostrado limitaciones técnicas en otros bacilos Gram negativos como es el caso de Pseudomonas aeruginosa [30-33].

Otra prueba fenotípica empleada para la detección de carbapenemasas tanto en miembros de la familia Enterobacteriaceae como en bacilos Gram negativos no fermentadores es el test tridimensional (3D), una adaptación del test de Hodge modificado, que ha sido empleado tradicionalmente para la detección de las $\beta$-lactamasas AmpC [34-36] y ha sido adaptado para la detección de carbapenemasas [37]. En el test 3D las bacterias productoras de carbapenemasas son lisadas, ya sea por acción mecánica, mediante sonicación o por ciclos de congelacióndescongelación para liberar las enzimas al medio [34,36]. No obstante, esta prueba no ha sido validada por el CLSI y su implementación de rutina en los laboratorios es cuestionable debido a su procedimiento laborioso.

En Colombia Pseudomonas aeruginosa se encuentra dentro de los cinco microorganismos más frecuentemente aislados y presenta una resistencia a carbapenémicos que oscila entre el $21,3 \%$ y el $26,2 \%$, incluso puede alcanzar hasta un $36 \%$ en las unidades de cuidados intensivos [38]. De manera preocupante, las cepas de Pseudomonas aeruginosa productoras de carbapenemasas han emergido y se han diseminado a varias regiones del país [39-44]. Inicialmente se reportaron las carbapenemasas de tipo VIM [42,43], que en la actualidad son las de mayor frecuencia [39,44]; posteriormente, en 2007, se informó por primera vez en el mundo la presencia de KPC en Pseudomonas aeruginosa en un aislado proveniente de la ciudad de Medellín [4I]. Debido a que Colombia es una región endémica para organismos productores de KPC [45-49] esta carbapenemasa ha venido en aumento y ha llegado a encontrarse en proporciones similares a VIM $[39,40,50]$ e incluso se han reportado con relativa frecuencia cepas que coproducen VIM y KPC $[39,40,44]$. Por otro lado, se han detectado las carbapenemasas NDM y GES en una menor frecuencia $[39,44]$.

En este contexto la ausencia de métodos fenotípicos aprobados por el CLSI y que sean de fácil implementación para su uso de rutina en los laboratorios de Microbiología Clínica genera limitaciones importantes en el diagnóstico y el control de las infecciones [30-33]. Teniendo en cuenta lo anterior, el presente estudio tuvo como objetivo evaluar tres variaciones del test de Hodge modificado previamente descritas en la literatura para la detección de carbapenemasas 
en una colección de aislados de Pseudomonas aeruginosa resistentes a carbapenémicos, recolectadas durante los años 2012 y 2014 en varias instituciones de la ciudad de Medellín, Colombia.

\section{Materiales y métodos}

\section{Aislados bacterianos}

El estudio incluyó 27 aislados de Pseudomonas aeruginosa con sensibilidad intermedia y resistencia a los carbapénemicos de acuerdo con los criterios del CLSI (20I2), seleccionados por conveniencia a partir de una colección recolectada durante un estudio previo realizado en instituciones de Medellín (Colombia) entre 2012 y 20I4. La selección se dio con base en el valor de la concentración inhibitoria mínima (CIM) encontrada a los carbapenémicos ertapenem, imipenem y meropenem, y a la presencia de los genes de carbapenemasas en cada aislado.

\section{Identificación fenotípica de carbapenemasas \\ Test tridimensional}

Se realizó el protocolo previamente descrito por Correa y colaboradores (2008) [37] con algunas modificaciones. Brevemente, los extractos bacterianos fueron obtenidos por lisis mecánica a través de la mezcla con micropipeta por mínimo 60 veces de una suspensión bacteriana con un inóculo alto, seguido de una centrifugación a 14.000 rpm durante 30 minutos y posterior recuperación del sobrenadante. Luego, el extracto fue depositado en el agar en una hendidura lineal (aproximadamente de $3 \mathrm{~cm}$ ) realizada mediante corte con una cuchilla quirúrgica estéril a $5 \mathrm{~mm}$ del borde del disco de carbapenémico. Se consideró un resultado positivo (sugestivo de presencia de carbapenemasas) cuando se observó deformidad o achatamiento en el halo de inhibición alrededor del carbapenémico.

\section{Test de Hodge modificado}

Se realizaron tres variaciones del protocolo recomendado por el CLSI [29]:

$\rightarrow$ La primera variación fue descrita inicialmente por Lee y colaboradores (20 I0) [5 I] y consistió en emplear agar MacConkey en lugar de Mueller Hinton para favorecer la liberación de las carbapenemasas de las células en presencia de compuestos biliares.

$\rightarrow$ La segunda variación fue descrita inicialmente por Pasteran y colaboradores (201 I) [33] y consistió en emplear Klebsiella pneumoniae ATCC 700603 como cepa indicadora sensible en lugar de Escherichia coli ATCC 25922, ya que se han reportado resultados equívocos o indeterminados debidos a la inhibición de Escherichia coli por algunas cepas de Pseudomonas aeruginosa [32].

$\rightarrow$ La tercera variación consistió en emplear las dos modificaciones previamente mencionadas simultáneamente.

Para todos los ensayos se empleó como control positivo la cepa Klebsiella pneumoniae ATCC I 705 y la cepa de Pseudomonas aeruginosa multisensible PAO-I como control negativo. Las pruebas se interpretaron como positivas (productoras de carbapenemasas) cuando la cepa indicadora mostró distorsión o achatamiento en el halo de inhibición alrededor del carbapenémico. 


\section{Identificación molecular de carbapenemasas}

La detección molecular de las carbapenemasas se llevó a cabo mediante la reacción en cadena de la polimerasa (PCR; del inglés, Polymerase Chain Reaction) múltiple que permite la detección simultánea de los genes de las carbapenemasas KPC $\left(b_{1} a_{\mathrm{KPC}}\right)$ VIM $\left(b / a_{\mathrm{VIM}}\right)$, IMP $\left(b / a_{\mid M P}\right), N D M\left(b / a_{N D M}\right)$, y OXA-48 (bla $\left.{ }_{\text {OXA-48 }}\right)$, mediante protocolos previamente descritos y validados $[23,25]$.

\section{Análisis estadístico}

Se calculó la sensibilidad, especificidad y valores predictivos negativo y positivo de las diferentes variaciones del test de Hodge modificado tomando como prueba de referencia para las comparaciones la reacción en cadena de la polimerasa para la detección molecular de las carbapenemasas. El análisis fue llevado a cabo en el software estadístico EPIDAT versión 3.I (Dirección Xeral de Innovación e Xestión da Saúde Pública, Xunta de Galicia-Organización Panamericana de la Salud).

\section{Resultados}

\section{Concentración mínima inhibitoria a carbapenémicos y producción de carbapanemasas}

De las 27 cepas de Pseudomonas aeruginosa incluidas, I I (40,7\%) presentaban una concentración inhibitoria mínima mayor o igual que 16 tanto para el imipenem como para el meropenem, nueve (33,3\%) tenían una concentración inhibitoria mínima mayor o igual que 16 para el imipenem y menor o igual que 8 para el meropenem, dos (7,4\%) presentaban concentración inhibitoria mínima menor o igual que 8 para imipenem y mayor o igual que 16 para meropenem, y las cinco (18,5\%) restantes presentaban una concentración inhibitoria mínima menor o igual que 8 para ambos carabapenémicos (véase tabla I).

Respecto a las características moleculares, 20 (74, 1\%) aislados fueron productores de carbapenemasas, en mayor frecuencia del tipo VIM (I4/20; 70,0\%) que de las KPC (8/20; 40,0\%), resaltando que de estos dos fueron positivos simultáneamente para ambas enzimas. Los siete $(25,9 \%)$ aislados restantes no fueron productores de estas enzimas según los hallazgos mediante la reacción en cadena de la polimerasa (véase tabla I). En cuanto a las demás carbapenemasas no fueron detectadas en ninguno de los aislados.

\section{Test tridimensional}

De los 27 aislados de Pseudomonas aeruginosa incluidos en el estudio, 17 (63,0\%) fueron positivos mediante el test 3D. De los 20 productores de carbapenemasas detectados molecularmente, 15 $(75,0 \%)$ fueron identificados como positivos mediante el test 3D (verdaderos positivos) y cinco $(25,0 \%)$ como negativos (falsos negativos). Entretanto, de los siete aislados que no presentaron genes de carbapenemasas se identificaron cinco $(71,4 \%)$ como negativos (verdaderos negativos) y dos $(28,6 \%)$ como positivos (falsos positivos) mediante el test 3D. Los dos aislados que presentaron simultáneamente las carbapenemasas KPC y VIM en las pruebas moleculares fueron detectadas satisfactoriamente como positivas mediante el test 3D (véase tabla I). 


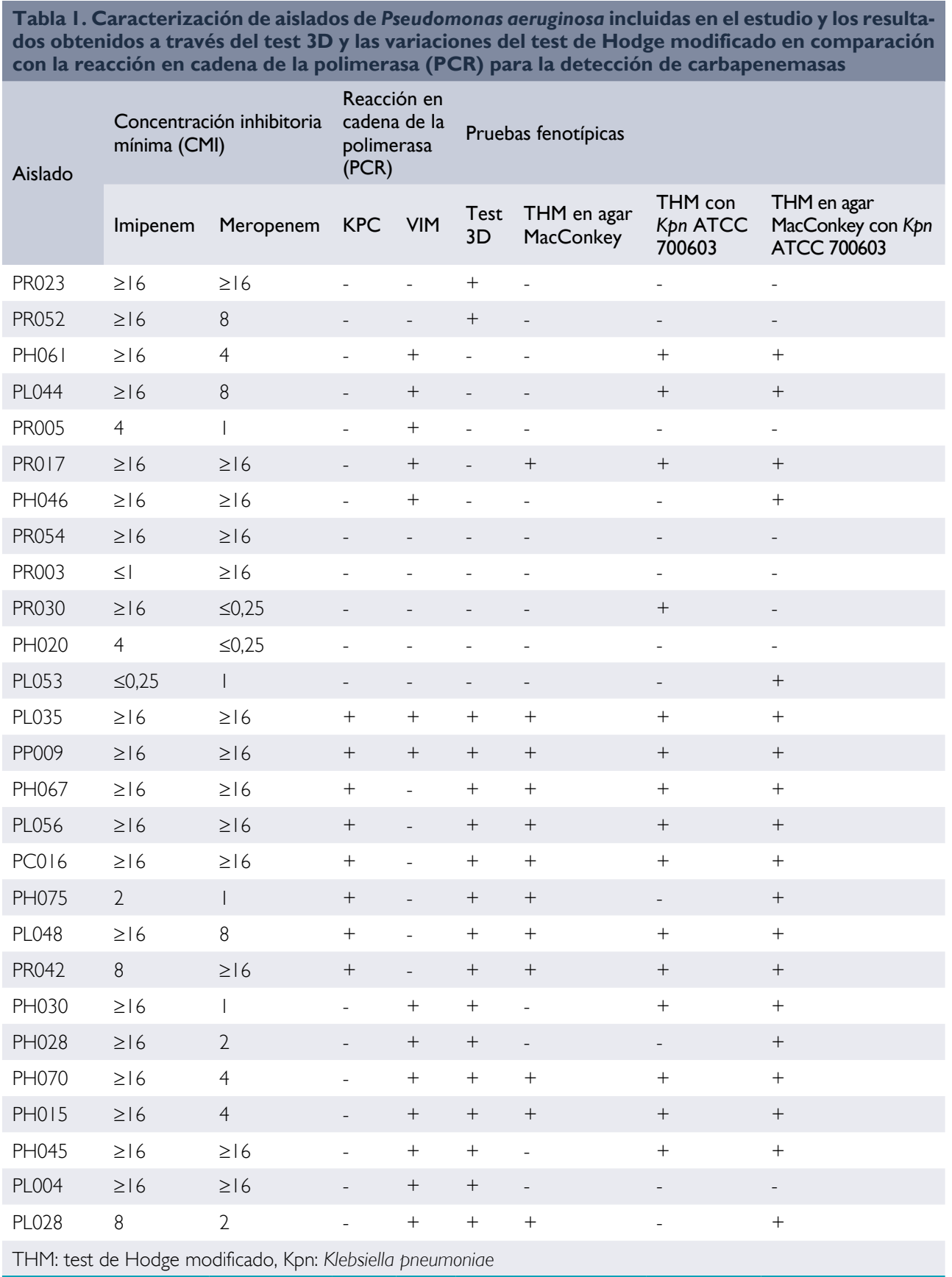

De manera global, el test tridimensional presentó una sensibilidad del 75,0\% y una especificidad del 7 I,4\%, un valor predictivo positivo del $88,2 \%$ y un valor predictivo negativo del $50,0 \%$ (véase tabla 2). Así mismo, el porcentaje de aislamientos correctamente clasificados como positivos y como negativos por el test 3D fue del $74,1 \%$, lo que sugiere que tiene una baja utilidad clínica. 


\begin{tabular}{|c|c|c|c|c|}
\hline Método & Sensibilidad (\%) & $\begin{array}{l}\text { Especificidad } \\
(\%)\end{array}$ & $\begin{array}{l}\text { Valor predicti- } \\
\text { vo positivo (\%) }\end{array}$ & $\begin{array}{l}\text { Valor predicti- } \\
\text { vo negativo (\%) }\end{array}$ \\
\hline Test tridimensional (3D) & 75,0 & 71,4 & 88,2 & 50,0 \\
\hline $\begin{array}{l}\text { Test de Hodge modificado en agar } \\
\text { MacConkey }\end{array}$ & 60,0 & 100,0 & 100,0 & 46,7 \\
\hline $\begin{array}{l}\text { Test de Hodge modificado con Kleb- } \\
\text { siella pneumoniae ATCC } 700603\end{array}$ & 70,0 & 85,7 & 93,3 & 50,0 \\
\hline $\begin{array}{l}\text { Test de Hodge modificado en agar } \\
\text { MacConkey con Klebsiella pneumoniae } \\
\text { ATCC } 700603\end{array}$ & 90,0 & 85,7 & 94,7 & 75,0 \\
\hline
\end{tabular}

\section{Variaciones al Test de Hodge Modificado para la detección de carbapenemasas}

\section{Test de Hodge modificado empleando agar MacConkey}

De los 27 aislados de Pseudomonas aeruginosa incluidos en el estudio, 12 (44,4\%) fueron positivos mediante el test de Hodge modificado empleando agar MacConkey (véase figura I). De las 20 cepas productoras de carbapenemasas por la reacción en cadena de la polimerasa, 12 (60,0\%) fueron positivas por este método (verdaderos positivos) y ocho $(40,0 \%)$ negativas (falsos negativos). Las siete cepas que no presentaron ninguna de las carbapenemasas por el método molecular fueron negativas por este método (verdaderos negativos), por consiguiente, no se obtuvo ningún resultado falso positivo. Es importante mencionar que los dos aislados productores de las carbapenemasas KPC y VIM simultáneamente también fueron positivos en esta variación del test de Hodge modificado (véase tabla I).

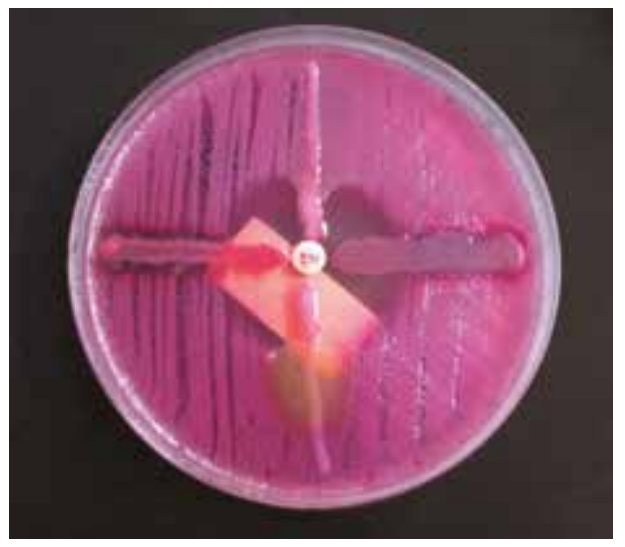

Figura I. Test de Hodge modificado empleando agar MacConkey. La estría superior vertical corresponde al control positivo (Klebsiella pneumoniae I705), la estría inferior vertical al control negativo (Pseudomonas aeruginosa multisensible, cepa PAO-I), las estrías horizontales corresponden a cepas problema de Pseudomonas aeruginosa. Obsérvese que el aislado a la izquierda presentó un resultado positivo y el de la derecha negativo para la producción de carbapenemasas.

En total, la sensibilidad y la especificidad para la variación del test de Hodge modificado empleando agar MacConkey fue del 60,0\% y el 100,0\%, respectivamente. En cuanto a la utilidad clínica se encontró un valor predictivo positivo y negativo del $100,0 \%$ y el $46,7 \%$, respectivamente. Además, el porcentaje de aislamientos correctamente clasificados, tanto como positivos así como negativos, con esta variación fue del 70,4\% (véase tabla 2). 


\section{Test de Hodge modificado empleando como cepa indicadora Klebsiella pneumoniae ATCC 700603}

De los 27 aislados de Pseudomonas aeruginosa incluidos en el estudio, I 5 (55,6\%) fueron positivos mediante el test de Hodge modificado empleando como cepa indicadora Klebsiella pneumoniae ATCC 700603 (véase figura 2). De las 20 cepas productoras de carbapenemasas mediante la reacción en cadena de la polimerasa 14 (70,0\%) fueron positivas por este ensayo (verdaderos positivos) y seis (30\%) fueron negativas (falsos negativos). De las siete cepas negativas para los genes de las carbapenemasas seis $(85,7 \%)$ fueron resultados verdaderos negativos y uno (14,3\%) falso positivo en esta segunda variación del test de Hodge modificado. Es relevante mencionar que los resultados para las dos cepas coproductoras de KPC Y VIM mostraron positividad aplicando esta variación (véase tabla I).

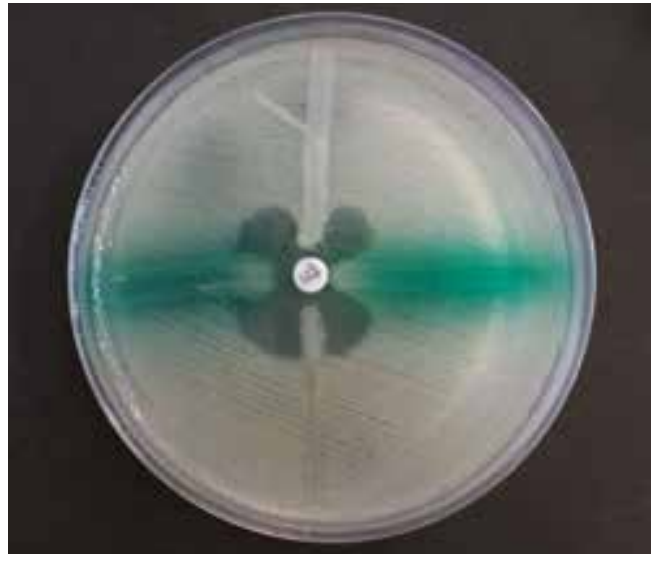

Figura 2. Test de Hodge modificado empleando la cepa indicadora sensible Klebsiella pneumoniae ATCC 700603. La estría vertical superior corresponde al control positivo (Klebsiella pneumoniae 1705), la estría vertical inferior al control negativo (Pseudomonas aeruginosa multisensible, cepa PAO-I) y las estrías horizontales corresponden a dos cepas problema de Pseudomonas aeruginosa. Obsérvese que el aislado a la izquierda presentó un resultado negativo para la producción de carbapenemasas y el aislado de la derecha positivo.

En esta segunda variación del test de Hodge modificado se encontró una sensibilidad del $70,0 \%$ y una especificidad del $85,7 \%$. La utilidad clínica para esta variación mostró un valor predictivo positivo del $93,3 \%$ y un valor predictivo negativo del 50,0\%. Así mismo, el 74, I\% de los aislamientos fueron correctamente clasificados como negativos y como positivos por esta variación (véase tabla 2).

\section{Test de Hodge modificado con la cepa indicadora sensible Klebsiella pneumoniae ATCC 700603 en agar MacConkey}

De los 27 aislados de Pseudomonas aeruginosa incluidos en el estudio, 19 (70,4\%) fueron positivos mediante el test de Hodge modificado empleando como cepa indicadora Klebsiella pneumoniae ATCC 700603 en agar MacConkey (véase figura 3). De los 20 aislados positivos para carbapenemasas mediante la reacción en cadena la polimerasa I $8(90,0 \%)$ fueron positivos por esta variación del test de Hodge (verdaderos positivos) y dos ( $10,0 \%)$ fueron negativos (falsos negativos). Entretanto, de las siete cepas negativas para carbapenemasas por el método molecular seis (85,7\%) fueron negativas para este ensayo y uno (14,3\%) fue un falso positivo. Finalmente, al igual que con las variaciones anteriores, las dos cepas que presentaban simultáneamente las enzimas KPC y VIM también fueron positivas (véase tabla I). 

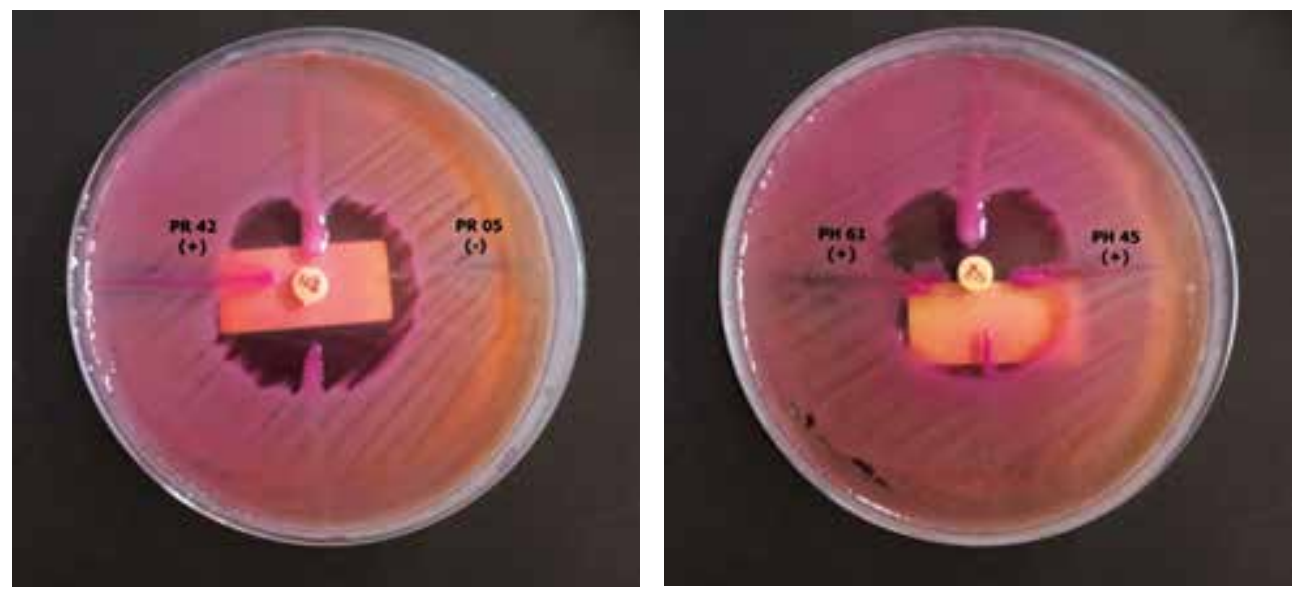

Figura 3. Test de Hodge modificado con agar MacConkey usando como cepa indicadora Klebsiella pneumoniae ATCC 700603. La estría vertical superior corresponde al control positivo (Klebsiella pneumoniae I705), la estría vertical inferior al control negativo (Pseudomonas aeruginosa multisensible, cepa PAO-I) y las estrías laterales corresponden a cepas problema de Pseudomonas aeruginosa. Obsérvese que los aislados PR042 (PR 42 (+)), PH06I (PH 6I (+)) y PH045 (PH 45 (+) presentaron un resultado positivo para la producción de carbapenemasas, mientras que el aislado PR005 (PR 05 (-)) fue negativo.

Esta variación del test de Hodge modificado empleando Klebsiella pneumoniae ATCC 700603 como cepa indicadora en agar MacConkey mostró una sensibilidad del 90,0\% y una especificidad del $85,7 \%$. Con relación a la validez clínica de este ensayo, se observaron valores predictivos positivo y negativo del $94,7 \%$ y $75,0 \%$. De igual manera, el porcentaje de aislados correctamente clasificados como negativos y como positivos fue del $88,9 \%$.

\section{Discusión}

Uno de los mecanismos de resistencia a los antibióticos más importantes en Pseudomonas aeruginosa es la producción de carbapenamasas; sin embargo, en la actualidad no existe un método fenotípico para su detección que se pueda emplear de rutina en los laboratorios de Microbiología Clínica y se encuentre avalado por el CLSI. En este sentido, es de gran relevancia la búsqueda de métodos fenotípicos accesibles que permitan brindarle al personal clínico herramientas que apoyen la toma de decisiones terapéuticas y de aislamiento, y a su vez, a minimizar los períodos de hospitalización y la carga económica que representan las infecciones asociadas a la atención en salud por microorganismos productores de carbapenemasas.

En este trabajo se propuso evaluar diferentes alternativas de pruebas fenotípicas para la detección de carbapenamasas en Pseudomonas aeruginosa. De manera general se puede decir que el test 3D, a pesar de poseer limitaciones, puede ser una alternativa para la detección fenotípica de carbapenemasas en Pseudomonas aeruginosa. Al contrastar los resultados obtenidos por el test tridimensional con diferentes variaciones del test de Hodge modificado se observó que la variación I, utilizando agar MacConkey, muestra una menor sensibilidad (60,0\% frente a 75,0\%), pero su especificidad es superior ( 100\% frente a 71,4\%). En cuanto a la utilidad clínica, el valor predictivo positivo de la variación I del test de Hodge modificado fue superior al del test 3D (I $00 \%$ frente a 88,2\%). Esto indica que un resultado positivo obtenido por este ensayo 
confirma la presencia de carbapenemasas; sin embargo, esto no se podría establecer con el test 3D. En cuanto a los valores predictivos negativos no se presentaron diferencias mayores, siendo bajo para ambas pruebas este valor (46,7\% para el test de Hodge modificado empleando agar MacConkey y 50\% para el test 3D), por lo tanto, un resultado negativo no descartaría la probabilidad de que la cepa problema, en realidad, sea productora de estas enzimas.

Al comparar la sensibilidad de la segunda variación del test de Hodge modificado, empleando como cepa indicadora Klebsiella pneumoniae ATCC 700603, con el test 3D se observa que no difiere sustancialmente (70,0\% frente a 75,0\%), mientras que para la especificidad esta variación presentó una mejoría (85,7\% frente a 7 I,4\%). Por otra parte, se encontró que un resultado positivo obtenido por esta segunda variación es más confiable que un resultado positivo obtenido por el test 3D, puesto que el valor predictivo positivo fue superior al del 3D (93,3\% frente a 88,2\%). Además, con esta segunda variación del test de Hodge modificado se encontró un aumento de la sensibilidad con respecto a la variación I (70,0\% frente a 60,0\%), aunque continúa siendo insuficiente para alcanzar la validez clínica. Sumado a esto, la especificidad se vio disminuida respecto a la primera variación (85,7\% frente a I00\%). Además, la utilidad clínica de esta segunda variación mostró que, a pesar de que los resultados positivos son confiables, los resultados negativos siguen siendo dudosos (valor predictivo positivo del 93,3\% y valor predictivo negativo del 50,0\%).

En cuanto a la tercera variación del test de Hodge modificado, empleando agar MacConkey y Klebsiella pneumoniae ATCC 700603 como cepa indicadora, se evidenció un valor de sensibilidad superior (90,0\%) respecto a los ensayos anteriores (variación I del 60,0\% y variación 2 del 70,0\%); sin embargo, aunque su especificidad (85,7\%) fue igual a la segunda variación, se vio disminuida con respecto a la variación I (100,0\%), lo que indica que esta tercera variación detecta adecuadamente los verdaderos positivos; sin embargo, aún sigue generando un porcentaje bajo de falsos positivos ( 14,3\%). Con relación a la validez clínica de este ensayo de acuerdo a los resultados observados se puede inferir que los resultados positivos de la prueba son más confiables que los negativos (valores predictivos positivo y negativo del $94,7 \%$ y $75,0 \%$, respectivamente).

A pesar de que el test 3D se ha considerado como una prueba más sensible para la detección de carbapenemasas debido a que emplea extractos celulares que mejoran la difusión de las enzimas al medio, según los resultados encontrados en este estudio al compararlo con la tercera variación del test de Hodge modificado (agar MacConkey y Klebsiella pneumoniae ATCC 700603 como cepa indicadora), es notable la superioridad de esta variación frente al test 3D, presentando mayor sensibilidad (90,0\% frente a 75,0\%) y especificidad (85,7\% frente a $71,4 \%)$. Igualmente, al establecer las diferencias de los valores predictivos positivo y negativo para ambas pruebas se muestran valores más altos para esta variación del test de Hodge $(94,7 \%$ y $75,0 \%$ frente a $88,2 \%$ y $50,0 \%$ ).

En resumen, de los ensayos realizados, la tercera variación fue la que mostró mejores resultados al presentar una sensibilidad superior a las demás variaciones (90\%), detectando de manera satisfactoria un número mayor de aislados verdaderos positivos para la producción de carbapenemasas; no obstante, se deben realizar más estudios que confirmen la reproducibilidad de esta tercera variación y su posible aplicabilidad en la práctica clínica. 
Con relación a la validez clínica que presentaban las diferentes variaciones del test de Hodge modificado se puede decir que a pesar de que la tercera variación mostró un valor predictivo positivo muy satisfactorio (94,7\%), su validez decae con respecto al valor predictivo negativo (75,0\%), por lo que se puede concluir que el método tiene dificultades para detectar los aislados verdaderos negativos para la producción de carbapenemasas. Sin embargo, dado que presentó los valores predictivos positivos y negativos más satisfactorios, se puede considerar como la mejor opción para su uso clínico entre las variaciones evaluadas.

Por otro lado, se pudo observar que las cepas que fueron detectadas como productoras de carbapenemasa tipo KPC mediante la reacción en cadena de la polimerasa, fueron detectadas en mayor proporción como positivas mediante las diferentes variaciones del test de Hodge modificado y el test 3D respecto a las cepas que portaban la carbapenemasa VIM. Estos resultados coinciden con estudios previos, donde se ha demostrado que la carbapenemasa tipo KPC presenta una actividad hidrolítica mayor que la exhibida por la carbapenemasa tipo VIM [19,52,53], por lo que es más fácil su detección in vitro.

Además de las variaciones del test de Hodge modificado propuestas se pueden emplear algunas pruebas complementarias para la detección de carbapenemasas, entre las que se encuentran las pruebas de sinergia con EDTA y con ácido borónico para la detección de carbapenemasas tipo metalobectalactamasas y serincarbapenemasas, respectivamente, con el fin de obtener resultados más confiables y que a su vez permitan conocer el tipo de carbapenemasa implicada [54].

\section{Conclusiones}

A través de este estudio se demostró que el test de Hodge modificado realizado en agar MacConkey y usando como cepa indicadora Klebsiella pneumoniae ATCC 700603 es una buena alternativa para la detección de carbapenemasas ya que presenta una mayor sensibilidad respecto a los demás ensayos evaluados en este trabajo. Es de gran importancia la realización de investigaciones adicionales en un número mayor de muestras, donde se determine la reproducibilidad de la técnica, con el fin de que se pueda implementar como prueba de rutina en los laboratorios clínicos y, así, para apoyar la toma de decisiones terapéuticas y mejorar el control de las infecciones.

\section{Agradecimientos}

A las instituciones participantes en el estudio por el suministro de los aislamientos.

\section{Bibliografía}

I. Aloush V, Navon-Venezia S, Seigman-Igra Y, Cabili S, Carmeli Y. Multidrug-resistant Pseudomonas aeruginosa: risk factors and clinical impact. Antimicrob Agents Chemother 2006; 50: 43-48.

2. Hirsch EB, Tam VH. Impact of multidrug-resistant Pseudomonas aeruginosa infection on patient out- comes. Expert Rev Pharmacoecon Outcomes Res 20I0; 10: 44|-45।.

3. Driscoll JA, Brody SL, Kollef MH. The epidemiology, pathogenesis and treatment of Pseudomonas aeruginosa infections. Drugs 2007; 67: 35I-368. 
4. Pier G, Ramphal R. Pseudomonas aeruginosa. En: Mandell G, Bennett J, Dollin R, eds. Mandell, Douglas, and Bennett's Principles and Practice of Infectious Diseases. Vol. 2 (ed 7a). Nueva York, Estados Unidos: Elsevier; 2010: 2835-2859.

5. Kerr KG, Snelling AM. Pseudomonas aeruginosa: a formidable and ever-present adversary. J Hosp Infect 2009; 73: 338-344.

6. Lister PD, Wolter DJ, Hanson ND. Antibacterial-resistant Pseudomonas aeruginosa: clinical impact and complex regulation of chromosomally encoded resistance mechanisms. Clin Microbiol Rev 2009; 22: 582-610.

7. Strateva T, Yordanov D. Pseudomonas aeruginosa - a phenomenon of bacterial resistance. J Med Microbiol 2009; 58: I |33- I | 48.

8. Pena C, Suarez C, Tubau F, Dominguez A, Sora M, Pujol M, et al. Carbapenem-resistant Pseudomonas aeruginosa: factors influencing multidrug-resistant acquisition in non-critically ill patients. Eur J Clin Microbiol Infect Dis 2009; 28: 519-522.

9. Papp-Wallace KM, Endimiani A, Taracila MA, Bonomo RA. Carbapenems: past, present, and future. Antimicrob Agents Chemother 201 I; 55: 4943-4960.

10. Gutierrez O, Juan C, Cercenado E, Navarro F, Bouza E, Coll P, et al. Molecular epidemiology and mechanisms of carbapenem resistance in Pseudomonas aeruginosa isolates from Spanish hospitals. Antimicrob Agents Chemother 2007; 51: 43294335.

II. Jimeno A, Alcalde MM, Blazquez A. [Epidemic outbreak of Pseudomonas aeruginosa carbepenem-resistant producing metallo-beta-lactamase]. Rev Clin Esp 201 1; 21 1: |87-191.

12. Rodriguez-Martinez JM, Poirel L, Nordmann P Molecular epidemiology and mechanisms of carbapenem resistance in Pseudomonas aeruginosa. Antimicrob Agents Chemother 2009; 53: 47834788.

13. Sacha P, Wieczorek P, Hauschild T, Zorawski M, Olszanska D, Tryniszewska E. Metallo-beta-lactamases of Pseudomonas aeruginosa--a novel mechanism resistance to beta-lactam antibiotics. Folia Histochem Cytobiol 2008; 46: I37- 142.

14. Nicolau CJ, Oliver A. Carbapenemasas en especies del género Pseudomonas. Enferm Infecc Microbiol Clin 2010; 28: 19-28.

15. Samuelsen O, Toleman MA, Sundsfjord A, Rydberg J, Leegaard TM, Walder M, et al. Molecular epidemiology of metallo-beta-lactamase-producing Pseudomonas aeruginosa isolates from Norway and Sweden shows import of international clones and local clonal expansion. Antimicrob Agents Chemother 2010; 54: 346-352.

16. Tam VH, Chang KT, LaRocco MT, Schilling AN, McCauley SK, Poole K, et al. Prevalence, mechanisms, and risk factors of carbapenem resistance in bloodstream isolates of Pseudomonas aeruginosa. Diagn Microbiol Infect Dis 2007; 58: 309-3। 4.

17. Wang J, Zhou JY, Qu TT, Shen P, Wei ZQ, Yu YS, et al. Molecular epidemiology and mechanisms of carbapenem resistance in Pseudomonas aeruginosa isolates from Chinese hospitals. Int J Antimicrob Agents 2010; 35: 486-491.

18. Livermore DM, Woodford N. The beta-lactamase threat in Enterobacteriaceae, Pseudomonas and Acinetobacter. Trends Microbiol 2006; 14: 4I3420.

19. Queenan AM, Bush K. Carbapenemases: the versatile beta-lactamases. Clin Microbiol Rev 2007; 20: 440-458.

20. Yong D, Choi YS, Roh KH, Kim CK, Park YH, Yum $\mathrm{JH}$, et al. Increasing prevalence and diversity of metallo-beta-lactamases in Pseudomonas spp., Acinetobacter spp., and Enterobacteriaceae from Korea. Antimicrob Agents Chemother 2006; 50: | 884- | 886

21. Zhao WH, Hu ZQ. Beta-lactamases identified in clinical isolates of Pseudomonas aeruginosa. Crit Rev Microbiol 2010; 36: 245-258.

22. Diene SM, Rolain JM. Carbapenemase genes and genetic platforms in Gram-negative bacilli: Enterobacteriaceae, Pseudomonas and Acinetobacter species. Clin Microbiol Infect 2014; 20: 831-838.

23. Ellington MJ, Kistler J, Livermore DM, Woodford N. Multiplex PCR for rapid detection of genes encoding acquired metallo-beta-lactamases. I Antimicrob Chemother 2007; 59: 321 -322.

24. Miriagou V, Cornaglia G, Edelstein M, Galani I, Giske CG, Gniadkowski M, et al. Acquired carbapenemases in Gram-negative bacterial pathogens: detection and surveillance issues. Clin Microbiol Infect 2010; 16: I12-122.

25. Poirel L, Walsh TR, Cuvillier V, Nordmann P. Multiplex PCR for detection of acquired carbapenemase genes. Diagn Microbiol Infect Dis 2011; 70: 1 19-123.

26. Woodford N, Sundsfjord A. Molecular detection of antibiotic resistance: when and where? J Antimicrob Chemother 2005; 56: 259-26I.

27. Cohen Stuart J, Leverstein-Van Hall MA, Dutch Working Party on the Detection of Highly Resistant M. Guideline for phenotypic screening and confirmation of carbapenemases in Enterobacteriaceae. Int J Antimicrob Agents 20 I0; 36: 205-2I 0.

28. CDC. CRE Toolkit - Guidance for Control of Carbapenem-resistant Enterobacteriaceae (CRE) 2012. Centers for Disease Control and Prevention. 2012. Disponible: http://www.cdc.gov/hai/pdfs/ cre/CRE-guidance-508.pdf. Consultado: diciembre 2015.

29. CLSI. Performance Standards for Antimicrobial Susceptibility Testing: Twenty-sixth Informational Supplement. CLSI document MI00-S26. Pensilvania, 
Estados Unidos: Clinical and Laboratory Standards Institute; 2016.

30. Lee K, Chong Y, Shin HB, Kim YA, Yong D, Yum $\mathrm{JH}$. Modified Hodge and EDTA-disk synergy tests to screen metallo-beta-lactamase-producing strains of Pseudomonas and Acinetobacter species. Clin Microbiol Infect 200 I; 7: 88-91.

31. Lee K, Lim YS, Yong D, Yum JH, Chong Y. Evaluation of the Hodge test and the imipenem-EDTA double-disk synergy test for differentiating metallo-beta-lactamase-producing isolates of Pseudomonas spp. and Acinetobacter spp. J Clin Microbiol 2003; 41: 4623-4629.

32. Pasteran F, Veliz O, Faccone D, Guerriero L, Rapoport $M$, Mendez $T$, et al. A simple test for the detection of KPC and metallo-beta-lactamase carbapenemase-producing Pseudomonas aeruginosa isolates with the use of meropenem disks supplemented with aminophenylboronic acid, dipicolinic acid and cloxacillin. Clin Microbiol Infect 2011; 17: |438-|44|.

33. Pasteran F, Veliz O, Rapoport M, Guerriero L, Corso A. Sensitive and specific modified Hodge test for KPC and metallo-beta- lactamase detection in Pseudomonas aeruginosa by use of a novel indicator strain, Klebsiella pneumoniae ATCC 700603. J Clin Microbiol 20 I I; 49: 430 I-4303.

34. Coudron PE, Hanson ND, Climo MW. Occurrence of extended-spectrum and AmpC beta-lactamases in bloodstream isolates of Klebsiella pneumoniae: isolates harbor plasmid-mediated FOX-5 and ACT-I AmpC beta-lactamases. J Clin Microbiol 2003; 41: 772-777.

35. Manchanda V, Singh NP. Occurrence and detection of AmpC beta-lactamases among Gram-negative clinical isolates using a modified three-dimensional test at Guru Tegh Bahadur Hospital, Delhi, India. J Antimicrob Chemother 2003; 5I: 4I5-4I8.

36. Nasim K, Elsayed S, Pitout JD, Conly J, Church DL, Gregson DB. New method for laboratory detection of AmpC beta-lactamases in Escherichia coli and Klebsiella pneumoniae. J Clin Microbiol 2004; 42: 4799-4802.

37. Correa A, Guzmán AM, Reyes SL, Ospina D, Torres JA, Tafur JD, et al. Evaluation of the Three-Dimensional (3D) Test as a Screening Tool for the Detection of Carbapenemases in Pseudomonas aeruginosa $(\mathrm{Pa})$ and Acinetobacter baumannii $(\mathrm{Ab})$. 48th Annual Interscience Conference on Antimicrobial Agents and Chemotherapy ICAAC, October 25 - 28. Washington D.C., Estados Unidos: American Society for Microbiology; 2008.

38. Ovalle M, Beltrán M. Vigilancia de Resistencia bacteriana (Whonet) Primer semestre año 2015. Bogotá: Instituto Nacional de Salud, Dirección de Redes en Salud Pública. 2015. Disponible: http:// www.ins.gov.co/tramites-y-servicios/examenesde-inter\%C3\%A9s-en-salud-publica/Microbiologa/ Informe\%20de\%20resistencia\%20Whonet\%20\%20 1\%20semestre\%2020I5.pdf. Consultado: diciembre
2015.

39. Saavedra SY, Duarte C, Gonzalez MN, Realpe ME. [Characterization of isolates of carbapenemase-producing Pseudomonas aeruginosa from seven Colombian provinces]. Biomedica 2014; 34: 217-223.

40. Vanegas JM, Cienfuegos AV, Ocampo AM, Lopez L, del Corral H, Roncancio G, et al. Similar frequencies of Pseudomonas aeruginosa isolates producing KPC and VIM carbapenemases in diverse genetic clones at tertiary-care hospitals in Medellin, Colombia. J Clin Microbiol 20 I4; 52: 3978-3986.

4I. Villegas MV, Lolans K, Correa A, Kattan JN, Lopez JA, Quinn JP, et al. First identification of Pseudomonas aeruginosa isolates producing a KPC-type carbapenem-hydrolyzing beta-lactamase. Antimicrob Agents Chemother 2007; 5I: I553- 1555.

42. Villegas MV, Lolans $K$, del Rosario Olivera M, Suarez CJ, Correa A, Queenan AM, et al. First detection of metallo-beta-lactamase VIM-2 in Pseudomonas aeruginosa isolates from Colombia. Antimicrob Agents Chemother 2006; 50: 226-229.

43. Crespo MP, Woodford N, Sinclair A, Kaufmann ME, Turton J, Glover J, et al. Outbreak of carbapenem-resistant Pseudomonas aeruginosa producing VIM-8, a novel metallo-beta-lactamase, in a tertiary care center in Cali, Colombia. J Clin Microbiol 2004; 42: 5094-5101.

44. González M, Hidalgo A, Saavedra S, Duarte C. Caracterización fenotípica y genotípica de perfiles de resistencia antimicrobiana de aislamientos bacterianos recuperados en Infecciones Asociadas a la Atención en Salud (IAAS) 2012 -2014. Programa de resistencia antimicrobiana en IAAS, Grupo de Microbiología, Subdirección Laboratorio Nacional de Referencia, Dirección de Redes en Salud Pública, Instituto Nacional de Salud, Colombia. 2014. Disponible: http://www.ins.gov.co/tramites-y-servicios/ examenes-de-inter\%C3\%A9s-en-salud-publical Microbiologa/CARACTERIZACI\%C3\%93N\%20 F E N O T \% C $3 \% 8$ D P I C A \% 20 Y \% 20 GENOT\%C3\%8DPICA\%20DE\%20PERFILES\%20 DE\%20RESISTENCIA\%20ANTIMICROBIANA\%20ASOCIADAS\%20A\%20IAAS.pdf. Consultado: diciembre 2015.

45. Lopez JA, Correa A, Navon-Venezia S, Correa AL, Torres JA, Briceno DF, et al. Intercontinental spread from Israel to Colombia of a KPC-3-producing Klebsiella pneumoniae strain. Clin Microbiol Infect 201।; 17: 52-56.

46. Mojica MF, Correa A, Vargas DA, Maya JJ, Montealegre MC, Rojas LJ, et al. Molecular correlates of the spread of KPC-producing Enterobacteriaceae in Colombia. Int J Antimicrob Agents 2012; 40: 277279.

47. Pacheco R, Osorio L, Correa AM, Villegas MV. [Prevalence of Gram-negative bacteria harboring bla KPC gene in Colombian hospitals]. Biomedica 20। 4; 34: 81-90. 
48. Maya JJ, Ruiz SJ, Blanco VM, Gotuzzo E, GuzmanBlanco M, Labarca J, et al. Current status of carbapenemases in Latin America. Expert Rev Anti Infect Ther 20 I3; I I: 657-667.

49. Ocampo AM, Chen L, Cienfuegos AV, Roncancio G, Chavda KD, Kreiswirth BN, et al. A TwoYear Surveillance in Five Colombian Tertiary Care Hospitals Reveals High Frequency of Non-CG258 Clones of Carbapenem-Resistant Klebsiella pneumoniae with Distinct Clinical Characteristics. Antimicrob Agents Chemother 20 I6; 60: 332-342.

50. Cuzon G, Naas T, Villegas MV, Correa A, Quinn JP, Nordmann P. Wide dissemination of Pseudomonas aeruginosa producing beta-lactamase blaKPC-2 gene in Colombia. Antimicrob Agents Chemother 20II; 55: 5350-5353.

5I. Lee K, Kim CK, Yong D, Jeong SH, Yum JH, Seo $\mathrm{YH}$, et al. Improved performance of the modified
Hodge test with MacConkey agar for screening carbapenemase-producing Gram-negative bacilli. J Microbiol Methods 2010; 83: 149-152.

52. Queenan AM, Shang W, Flamm R, Bush K. Hydrolysis and inhibition profiles of beta-lactamases from molecular classes $A$ to $D$ with doripenem, imipenem, and meropenem. Antimicrob Agents Chemother 20।0; 54: 565-569.

53. Vasoo S, Cunningham SA, Kohner PC, Simner PJ, Mandrekar JN, Lolans K, et al. Comparison of a novel, rapid chromogenic biochemical assay, the Carba NP test, with the modified Hodge test for detection of carbapenemase-producing Gram-negative bacilli. J Clin Microbiol 20 I3; 5 I: 3097-3 I0 I.

54. Perozo A, Castellano M, Tutaya K, Toledo E, Arraiz N. Evaluación de métodos fenotípicos para la detección de metalobetalactamasas en aislados clínicos de Pseudomonas aeruginosa. Kasmera 2013; 4I: II5-126.

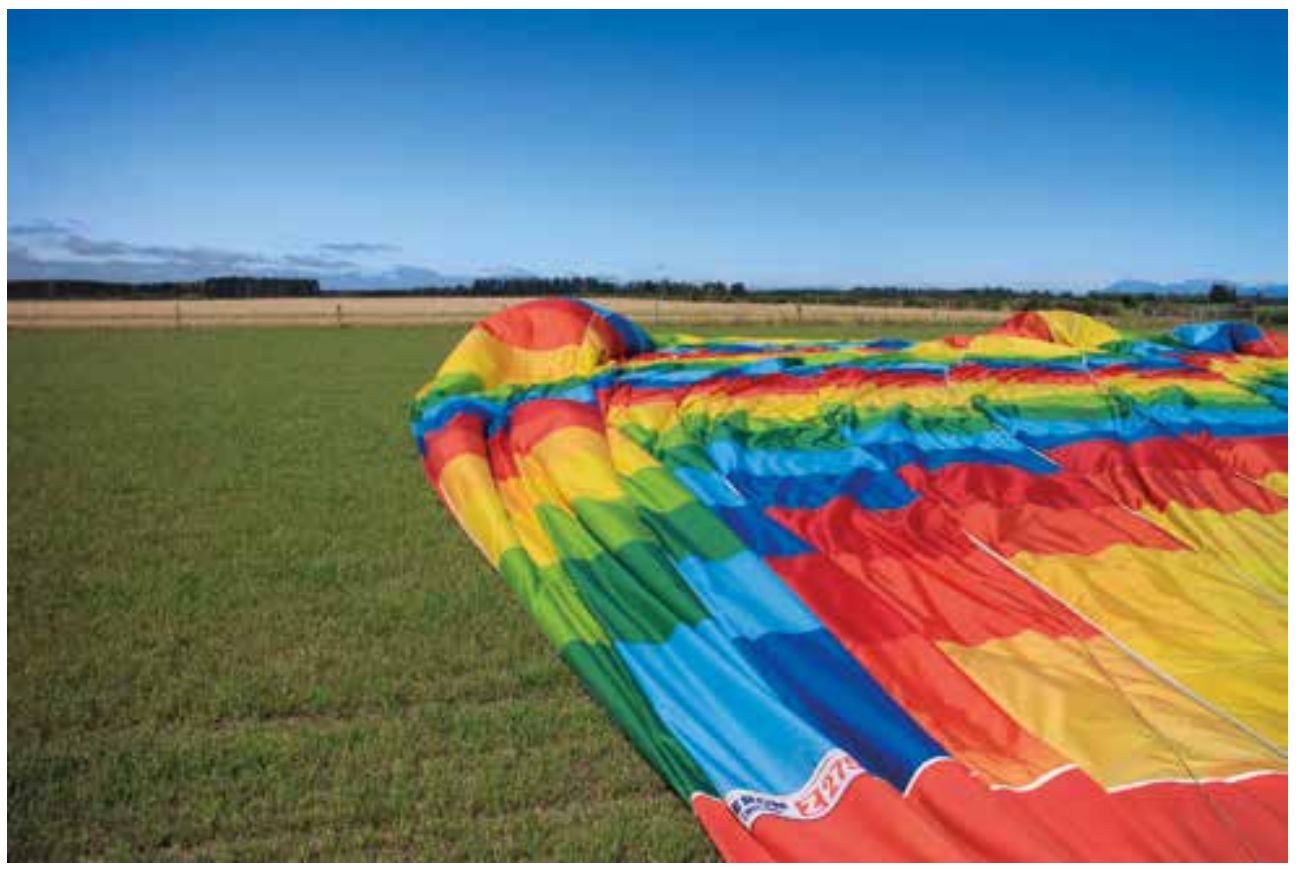

Vuelo en globo en Christchurch

Christchurch, New Zealand

Carlos Alberto Lozano M. carloslozano@iatm.com.co 